\title{
The Novel Formation of Barium Titanate Nanodendrites
}

\author{
Chien-Jung Huang, ${ }^{1}$ Kan-Lin Chen, ${ }^{2}$ Pin-Hsiang Chiu, ${ }^{3}$ Po-Wen Sze, \\ and Yeong-Her Wang ${ }^{3,5}$ \\ ${ }^{1}$ Department of Applied Physics, National University of Kaohsiung, Nanzih, Kaohsiung, Taiwan \\ ${ }^{2}$ Department of Electronic Engineering, Fortune Institute of Technology, Kaohsiung, Taiwan \\ ${ }^{3}$ Institute of Electro-Optical Science and Engineering, Institute of Microelectronics, National Cheng-Kung University, \\ Tainan, Taiwan \\ ${ }^{4}$ Department of Electro-Optical Science and Engineering, Kao Yuan University, Kaohsiung, Taiwan \\ ${ }^{5}$ Department of Electrical Engineering, Institute of Microelectronics, National Cheng-Kung University, Tainan, Taiwan
}

Correspondence should be addressed to Chien-Jung Huang; chien@nuk.edu.tw and Kan-Lin Chen; klchen@fotech.edu.tw

Received 15 March 2014; Accepted 26 March 2014; Published 30 April 2014

Academic Editor: Fu-Ken Liu

Copyright (c) 2014 Chien-Jung Huang et al. This is an open access article distributed under the Creative Commons Attribution License, which permits unrestricted use, distribution, and reproduction in any medium, provided the original work is properly cited.

The barium titanate $\left(\mathrm{BaTiO}_{3}\right)$ nanoparticles with novel dendrite-like structures have been successfully fabricated via a simple coprecipitation method, the so-called $\mathrm{BaTiO}_{3}$ nanodendrites (BTNDs). This method was remarkable, fast, simple, and scalable. The growth solution is prepared by barium chloride $\left(\mathrm{BaCl}_{2}\right)$, titanium tetrachloride $\left(\mathrm{TiCl}_{4}\right)$, and oxalic acid. The shape and size of $\mathrm{BaTiO}_{3}$ depend on the amount of added $\mathrm{BaCl}_{2}$ solvent. To investigate the influence of amount of $\mathrm{BaCl}_{2}$ on $\mathrm{BTNDs}$, the amount of $\mathrm{BaCl}_{2}$ was varied in the range from 3 to $6 \mathrm{~mL}$. The role of $\mathrm{BaCl}_{2}$ is found to have remarkable influence on the morphology, crystallite size, and formation of dendrite-like structures. The thickness and length of the central stem of BTND were $\sim 300 \mathrm{~nm}$ and $\sim 20 \mu \mathrm{m}$, respectively. The branchings were found to occur at irregular intervals along the main stem. Besides, the formation mechanism of BTND is proposed and discussed.

\section{Introduction}

Over the past few years, the unique ferroelectric, piezoelectric, and thermoelectric properties of barium titanate $\left(\mathrm{BaTiO}_{3}\right)$ nanoparticles have become increasingly important in the electronic ceramics industry. The $\mathrm{BaTiO}_{3}$ nanoparticles have been extensively applied in various fields such as multilayer ceramic capacitors (MLCCs), integral capacitors in printed circuit boards (PCB), dynamic random access memories (DRAM), resistors with positive temperature coefficient of resistivity (PTCR), temperature-humidity-gas sensors, electrooptic devices, piezoelectric transducers, actuators, and thermistors [1-9]. Among these applications, performance and characteristics are strongly influenced by size, shape, composition, morphology, spatial ordering, and impurities of the $\mathrm{BaTiO}_{3}$ nanoparticles. Thus, effectively controlling their shape and size is of high importance and is a challenging task for researchers and the industry. In this work, we have developed $\mathrm{BaTiO}_{3}$ with novel dendrite-like structures. Very recently, nanoparticles with dendrite-like structures have received much attention because of their potential application in device $[10,11]$. However, finely controlling the morphology of the $\mathrm{BaTiO}_{3}$ nanoparticles is extremely dependent on preparation method and synthesis procedure.

Traditionally, the $\mathrm{BaTiO}_{3}$ particle is prepared by the solidstate reaction method through heating $\mathrm{BaCO}_{3}$ and $\mathrm{TiO}_{2}$ at high temperature as $1200^{\circ} \mathrm{C}[12,13]$. The disadvantage of this method is that high calcinations temperature may strongly cause aggregation between the particles, and it takes a long time to produce submicrometer particles $(1 \sim 2 \mu \mathrm{m})$. Up to now, various new preparation methods have been developed and reported in fabricating $\mathrm{BaTiO}_{3}$ nanoparticles with high quality, well-controlled shape, and small size, such as the sol-gel method $[14,15]$, the hydrothermal method $[16,17]$, the Pechini processing using a citric or oxalate complex as the precursor $[18,19]$, the ball-milling method $[20,21]$, 
the polymeric precursor method [22], the soft chemical process [23], the glycolthermal method [24], and the coprecipitation method [25]. Among these, the coprecipitation method is superior to other methods in terms of the following characteristics: high growth rate, modest equipment, low processing temperature, ease of controlling the yield, low cost, large amount synthesized, and high quality [26].

In the coprecipitation method, the preparation of $\mathrm{BaTiO}_{3}$ nanoparticles through the coprecipitation of barium and titanium hydroxides from aqueous solutions has been reported since the early Flaschen research work [27]. Synthesis of $\mathrm{BaTiO}_{3}$ nanoparticles as the decomposition product of barium titanyl oxalate or barium titanyl citrate is a multistage process, depending on the gaseous medium, the dispersion of the starting reagents and intermediate phase (the degree of branching of the interphase surface), the regime in which the reaction occurs (kinetic or diffusion), the growth temperature, and the heating rate [28-32]. Although these previous studies succeeded in fabricating $\mathrm{BaTiO}_{3}$ nanoparticles, the procedure is quite complicated. Furthermore, these procedures also require special conditions, such as judicious choice of the stabilizer, heat treatment, and time duration. Therefore, it will be a significant challenge to simplify the procedure for the fabrication of $\mathrm{BaTiO}_{3}$ nanoparticles.

In our laboratory, we developed a simple procedure by slightly modifying the multistage process so it could be applied to fabricate $\mathrm{BaTiO}_{3}$ nanoparticles with wellcontrolled size. In this simple procedure, appropriate amount of stock solution of titanium tetrachloride $\left(\mathrm{TiCl}_{4}\right)$, barium chloride $\left(\mathrm{BaCl}_{2}\right)$, and oxalic acid was added in deionized water to form growth solution. The $\mathrm{BaTiO}_{3}$ nanoparticle was formed by coprecipitation of both barium and titanium precursor. During the coprecipitation process, titanium acted as the seed in the growth solution so that the barium could nucleate and precipitate onto the surfaces of titanium via the heterogeneous nucleation process. More importantly, it is found that the amount of added $\mathrm{BaCl}_{2}$ can be critical for shape and size of $\mathrm{BaTiO}_{3}$ nanoparticles.

In this study, we first reported the fabrication of $\mathrm{BaTiO}_{3}$ nanoparticles with novel dendrite-like structures through the coprecipitation method, the so-called $\mathrm{BaTiO}_{3}$ nanodendrites (BTNDs). It can be observed that the various amounts of added $\mathrm{BaCl}_{2}$ during nucleation and growth process caused the alteration of the $\mathrm{BaTiO}_{3}$ nanoparticles shape, forming the branch-like structures. Until now, to our knowledge, there are no reports yet on the synthesis of the BTNDs by coprecipitation method. A good understanding of the microstructure properties is a very important issue for the potential application of the BTNDs. Thus, a detailed model for the newly observed novel BTNDs is also proposed to explain their possible formation mechanism.

\section{Experimental Details}

Barium chloride $\left(\mathrm{BaCl}_{2} \cdot 2 \mathrm{H}_{2} \mathrm{O}, 99 \%\right)$ and oxalic acid $\left(\mathrm{C}_{2} \mathrm{H}_{2} \mathrm{O}_{4} \cdot 2 \mathrm{H}_{2} \mathrm{O}, 99 \%\right)$ were obtained from Riedel-deHaën (Sigma-Aldrich, USA). Titanium tetrachloride solution
$\left(\mathrm{TiCl}_{4}, 99 \%, 0.1 \mathrm{M}\right)$ was purchased from Fluka (SigmaAldrich, USA). All chemicals and materials were used without further purification. The distilled water used throughout the experiments was purified by a Milli-Q system (Millipore resistivity $18.2 \mathrm{M} \Omega \mathrm{cm}$ ). The BTNDs were fabricated by first dissolving $\mathrm{BaCl}_{2}$ in distilled water at 50 $70^{\circ} \mathrm{C}$. Separately, oxalic acid was dissolved in distilled water at $65^{\circ} \mathrm{C}$ in an ultrasonic tank with titanium tetrachloride slowly added. The two solutions were mixed in an ultrasonic bath at $65^{\circ} \mathrm{C}$. Nanometer-sized $\mathrm{BaTiO}_{3}$ particles were formed at this stage. Finally, the growth time was $20 \mathrm{~min}$.

The size and shape of the BTNDs were measured and analyzed by transmission electron microscopy (TEM, JEOL JEM1230 ) at an accelerating voltage of $80 \mathrm{kV}$. The microstructure of the BTNDs was observed by high-resolution transmission electron microscopy (HRTEM, Philips Tecnai G2 F20) with an accelerating voltage of $200 \mathrm{kV}$. The HRTEM was equipped with selected area electron diffraction (SAED) and an energydispersive X-ray (EDX) spectrometric element analyzer. The samples for TEM, SAED, and EDX were prepared by drop coating onto a standard 200-mesh, $3 \mathrm{~mm}$, carbon-coated copper grid (Agar Scientific, UK).

\section{Results and Discussion}

Figures 1(a)-1(d) show the TEM images of $\mathrm{BaTiO}_{3}$ nanoparticles obtained by adding $3,4,5$, and $6 \mathrm{~mL}$ of $\mathrm{BaCl}_{2}$. The results clearly show that the shape of the $\mathrm{BaTiO}_{3}$ nanoparticles can be changed by altering the amount of $\mathrm{BaCl}_{2}$. When the amount of $\mathrm{BaCl}_{2}$ was $3 \mathrm{~mL}$, the $\mathrm{BaTiO}_{3}$ nanoparticles with large quantities were almost spherical in shape and were small in size, as shown in Figure 1(a). The inset of Figure 1(a) shows the TEM image of $\mathrm{BaTiO}_{3}$ nanoparticles at higher magnification, indicating that the particle size is about $20 \mathrm{~nm}$. When the amount of $\mathrm{BaCl}_{2}$ was increased from 4 to $5 \mathrm{~mL}$, the shape of $\mathrm{BaTiO}_{3}$ nanoparticles began to change from spherical to dendrite-like, as shown in Figures 1(b) and 1(c). When the amount of $\mathrm{BaCl}_{2}$ was $6 \mathrm{~mL}$, the $\mathrm{BaTiO}_{3}$ nanoparticles were almost dendrite-like in shape, as shown in Figure 1(d). Even after sonication for TEM sample preparation, the branches of the dendrites were intact, indicating strong bonding between the grains. Thus, there is not any isolated spherical $\mathrm{BaTiO}_{3}$ particles in TEM image. However, the role of $\mathrm{BaCl}_{2}$ may be to act as shape-modifier to change $\mathrm{BaTiO}_{3}$ nanoparticles' shape from spherical to dendrite-like structure when the $\mathrm{BaCl}_{2}$ with high amount was added to growth solution during coprecipitation process. Besides, these results also show that the size of $\mathrm{BaTiO}_{3}$ nanoparticles increased as the amount of $\mathrm{BaCl}_{2}$ increased, as revealed TEM analysis (Figure 1).

Figure 2 shows the low-magnification TEM images of single BTND prepared with $6 \mathrm{~mL}$ of $\mathrm{BaCl}_{2}$. As can be seen in Figure 2(a), the BTND described as dendritic structures has a large area of several square micrometers. The thickness of the central stem of BTND was $\sim 300 \mathrm{~nm}$. Along the central stem (with length of $\sim 20 \mu \mathrm{m}$ ), branching was seen for every $\sim 300 \mathrm{~nm}$. The lengths of the side branches were found to be different for the same BTND. Also the angle between the main stem and the branch was not constant 
TABLE 1: Preparation of $\mathrm{BaTiO}_{3}$ particles using oxalate process.

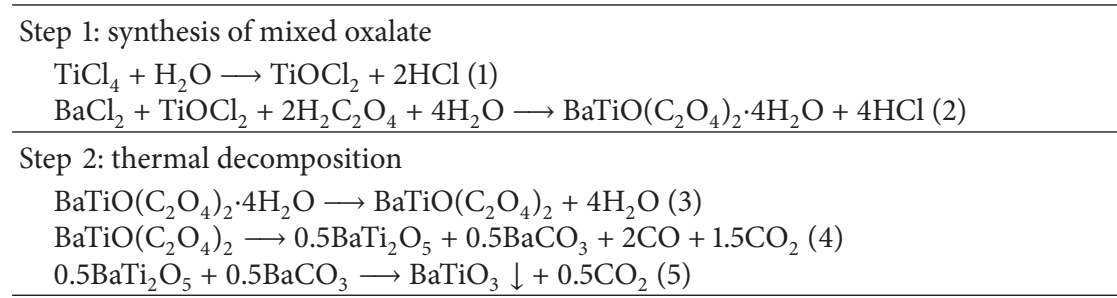

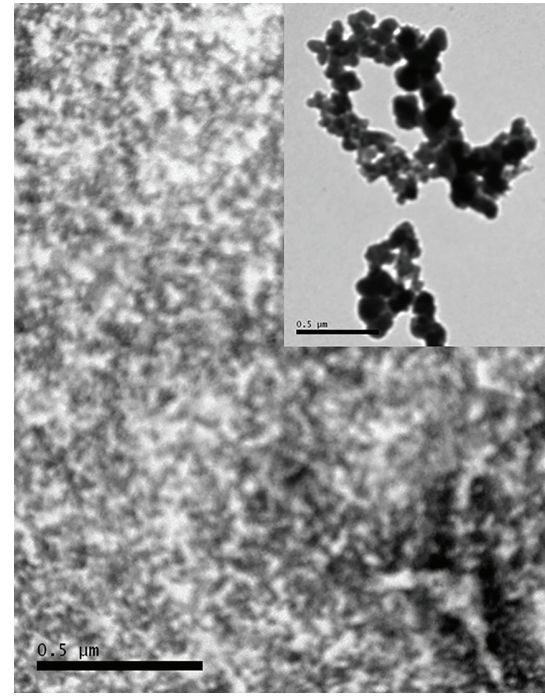

(a)

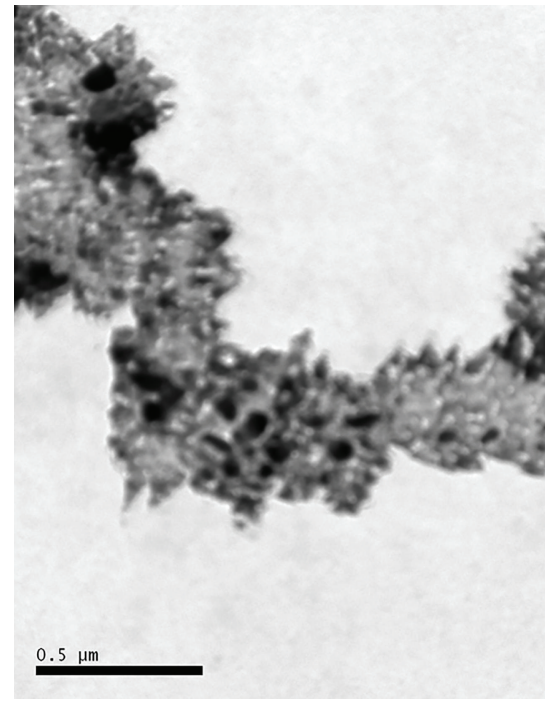

(c)



(b)

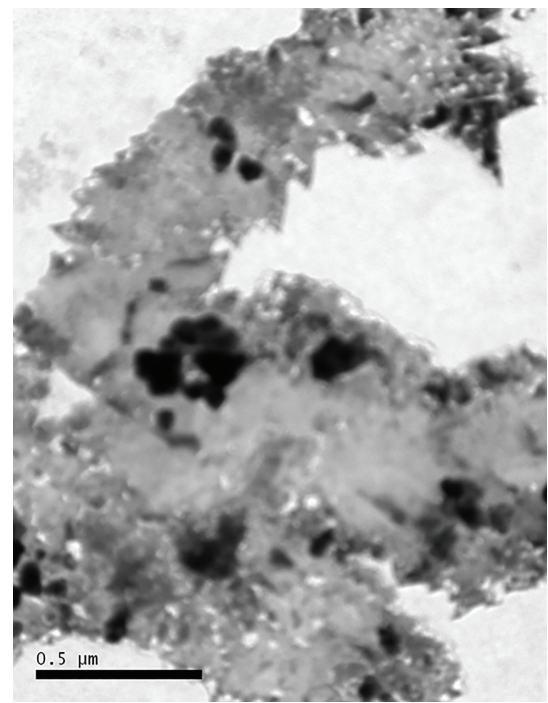

(d)

FIGURE 1: Transmission electron microscopy (TEM) images of the $\mathrm{BaTiO}_{3}$ nanoparticles prepared by (a) 3, (b) 4, (c) 5, and (d) $6 \mathrm{~mL}$ of barium chloride.

for all the cases, as shown in Figure 2(b). The aggregated crystallites may form a BTND by oriented attachment of the crystallites. The inset of Figure 2(b) shows the SAED pattern of the individual grain from the BTND. The characteristic ring in the polycrystalline diffraction pattern confirmed that the BTNDs are polycrystalline structures. Figure 2(c) shows high-magnification TEM image of stem of single BTND, which clearly shows that the dendrite-like structure consisted of eleven large $\mathrm{BaTiO}_{3}$ particles and many small $\mathrm{BaTiO}_{3}$ compounds between the particles. Figure 2(d) schematically 


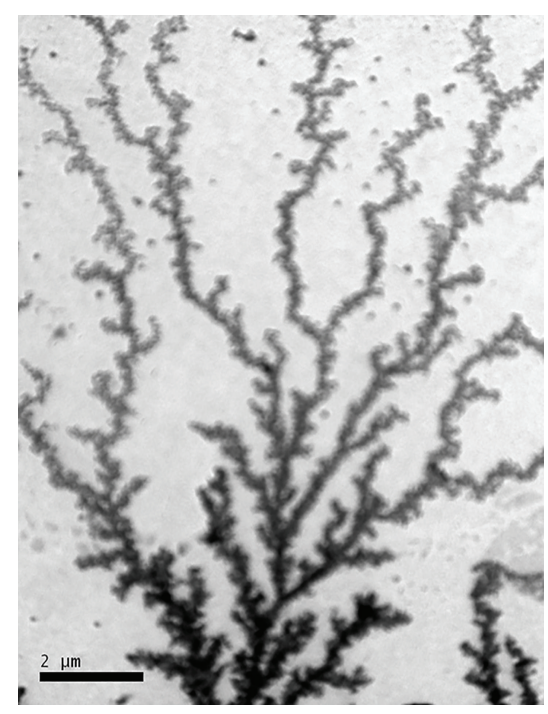

(a)

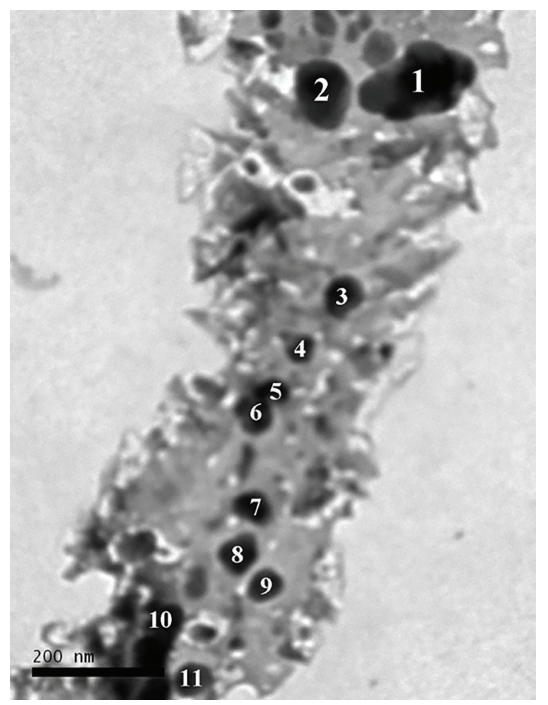

(c)



(b)

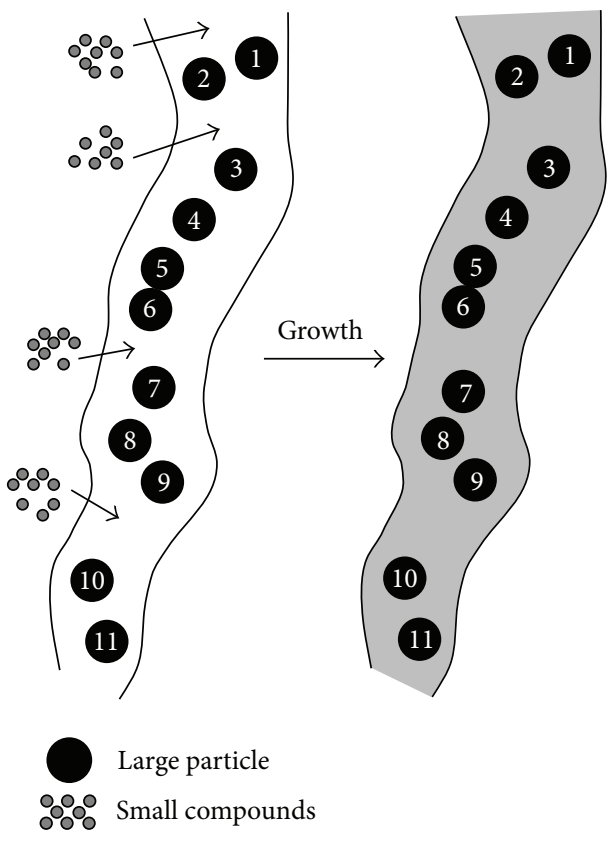

Formation of dendrite-like structures

(d)

FIGURE 2: TEM images of the BTND obtained by the oxalate coprecipitation method: (a) low-magnification image, (b) high-magnification image and SAED pattern, (c) the part of BTND at the stem, and (d) schematic illustration of formation of BTND.

shows the formation mechanism of BTNDs. The BTNDs were formed by aggregation of many small $\mathrm{BaTiO}_{3}$ compounds between the large $\mathrm{BaTiO}_{3}$ particles during the growth process, indicating that small $\mathrm{BaTiO}_{3}$ compounds linked the large $\mathrm{BaTiO}_{3}$ particles to form the dendrite-like shape. However, the present study is to show that the amount of $\mathrm{BaCl}_{2}$ is a key parameter in the formation of $\mathrm{BaTiO}_{3}$ nanoparticles with various sizes and shapes.

The $\mathrm{BaTiO}_{3}$ nanoparticles produced using the coprecipitation method were analyzed by using EDX for studying the composition of $\mathrm{BaTiO}_{3}$ nanoparticles, as shown in Figure 3.
The elements detected should be carbon, oxygen, titanium (Ti), and barium $(\mathrm{Ba})$ in the present method. No other elements were detected, indicating that the sample is purely $\mathrm{BaTiO}_{3}$. The peaks of copper $(\mathrm{Cu})$ and carbon in this chart correspond to the $\mathrm{Cu}$ grid coated with a thin carbon film as a carrier of the $\mathrm{BaTiO}_{3}$ nanoparticles during the test. The above findings support the hypothesis that the formation of BTNDs process is as follows. The relationship between the formation of BTNDs and the amount of $\mathrm{BaCl}_{2}$ can be easily explained through the chemical formation of $\mathrm{BaTiO}_{3}$ particles during oxalate process [33], as shown in Table 1. 




Figure 3: TEM image of single BTND and corresponding EDX spectra.

The precipitation of monodisperse $\mathrm{BaTiO}_{3}$ particles is generally formed with the synthesis of mixed oxalate (Step 1) and the thermal decomposition (Step 2). According to (1) of Step 1, Ti (IV) hydroxo complexes or Ti (IV) polyanions are produced by hydrolysis and condensation reactions. According to (2) of Step 1, starting materials $\mathrm{TiCl}_{4}$ and $\mathrm{BaCl}_{2}$ are reacted with water and oxalic acid $\left(\mathrm{H}_{2} \mathrm{C}_{2} \mathrm{O}_{4}\right)$ to precipitate a double oxalate $\left(\mathrm{BaTiO}\left(\mathrm{C}_{2} \mathrm{O}_{4}\right)_{2} \cdot 4 \mathrm{H}_{2} \mathrm{O}\right)$ precursor. This precursor was obtained by the reaction which proceeds in two steps: (i) initial rapid formation of a Ti-rich gel phase and (ii) slower reaction between the gel phase and the $\mathrm{Ba}^{2+}$ left in solution. According to Step 2, this precursor during growth process then results in formation of small $\mathrm{BaTiO}_{3}$ compounds (at atomic- or molecular-level compositional homogeneity) through thermal decomposition. Finally, the aggregation and the agglomeration of many small $\mathrm{BaTiO}_{3}$ compounds lead to the formation of crystalline $\mathrm{BaTiO}_{3}$ particle, and a white $\mathrm{BaTiO}_{3}$ particle precipitate can be readily observed. According to (2), the amount of double oxalate precursor is increased as the amount of $\mathrm{BaCl}_{2}$ increases when the $\mathrm{TiCl}_{4}$ is enough amounts. In other words, the amount of small $\mathrm{BaTiO}_{3}$ compounds is increased with the increase in amount of double oxalate precursor, as shown in Step 2 of Table 1. Thus, the aggregation of small $\mathrm{BaTiO}_{3}$ compounds is enhanced when the amount of small $\mathrm{BaTiO}_{3}$ compounds increases, resulting in the growth of $\mathrm{BaTiO}_{3}$ nanoparticles being enhanced and causing the size of the $\mathrm{BaTiO}_{3}$ nanoparticles to be increased. However, the size of $\mathrm{BaTiO}_{3}$ nanoparticles is directly proportioned to amount of $\mathrm{BaCl}_{2}$, with the results being consistent with TEM analysis of Figure 1.

In this study, we propose that the addition of $\mathrm{BaCl}_{2}$ causes the possible mechanism of BTNDs formation. It is found that a high amount of $\mathrm{BaCl}_{2}$ led to formation of large $\mathrm{BaTiO}_{3}$ particles and small $\mathrm{BaTiO}_{3}$ compounds during the coprecipitation growth that caused particle agglomeration to form BTNDs in the growth solution, as shown in Figure 2. The small $\mathrm{BaTiO}_{3}$ compounds aggregated onto the surface of the large $\mathrm{BaTiO}_{3}$ particles by the van der Waals attractions forces during growth process. It is considered to comprise mainly two processes: (i) the formation of small $\mathrm{BaTiO}_{3}$ compounds at the growth process and (ii) the subsequent anisotropic coalescence of these small $\mathrm{BaTiO}_{3}$ compounds leading to the BTNDs formation; that is to say, these small $\mathrm{BaTiO}_{3}$ compounds with an unstable state show a tendency to undergo fusion into dendrite-like structures. Hence, the amount of $\mathrm{BaCl}_{2}$ definitely has a critical role in the formation of the BTNDs. However, formation mechanism for BTNDs using the coprecipitation method via $\mathrm{BaCl}_{2}$ addition is still under investigation.

\section{Conclusions}

In summary, this study prepares polycrystalline BTNDs by a simple coprecipitation method. It has been observed that the amount of $\mathrm{BaCl}_{2}$ plays an important role in the formation of BTNDs. Change in the amount of $\mathrm{BaCl}_{2}$ from 3 to $6 \mathrm{~mL}$ strongly affected the shape of particles from sphere to dendrite-like shape. The formation of BTNDs was induced by aggregation of many small $\mathrm{BaTiO}_{3}$ compounds between the several large $\mathrm{BaTiO}_{3}$ particles during growth, causing the small $\mathrm{BaTiO}_{3}$ compounds to link to the large $\mathrm{BaTiO}_{3}$ particles forming dendrite-like structures. Further measurements are now necessary to get a better understanding of these BTNDs. This preparation of BTNDs is proven to be a simple and effective synthesis method.

\section{Conflict of Interests}

The authors declare that there is no conflict of interests regarding the publication of this paper.

\section{Acknowledgments}

This work was partially supported by the National Science Council of Taiwan (NSCT) under Contract no. NSC 1022221-E-390-019-MY2. The authors gratefully acknowledge the Southern Taiwan University of Technology (Taiwan) for the TEM measurement.

\section{References}

[1] U. van Stevendaal, K. Buse, S. Kämper, H. Hesse, and E. Krätzig, "Light-induced charge transport processes in photorefractive barium titanate doped with rhodium and iron," Applied Physics B: Lasers and Optics, vol. 63, no. 4, pp. 315-321, 1996.

[2] K. Kumar, "Ceramic capacitors: an overview," Electronics Information Planning, vol. 25, no. 11, pp. 559-582, 1998.

[3] J. F. Scott, "Status report on ferroelectric memory materials," Integrated Ferroelectrics, vol. 20, no. 1-4, pp. 15-23, 1998.

[4] A. B. Alles and V. I. Burdick, "Grain boundary oxidation in PTCR barium titanate thermistors," Journal of the American Ceramic Society, vol. 76, no. 2, pp. 401-408, 1993.

[5] Z. Zhi-Gang, Z. Gang, W. Ming, and Z. Zhong-Tai, "Temperauture-humidity-gas multifunctional sensitive ceramics," Sensors and Actuators, vol. 19, no. 1, pp. 71-81, 1989.

[6] M. Mori, T. Kineri, K. Kadono et al., "Effect of the atomic ratio of $\mathrm{Ba}$ to Ti on optical properties of gold-dispersed $\mathrm{BaTiO}_{3}$ thin films," Journal of the American Ceramic Society, vol. 78, no. 9, pp. 2391-2394, 1995. 
[7] H. Song, S. X. Dou, M. Chi, H. Gao, Y. Zhu, and P. Ye, "Studies of shallow levels in undoped and rhodium-doped barium titanate," Journal of the Optical Society of America B: Optical Physics, vol. 15, no. 4, pp. 1329-1334, 1998.

[8] C. Buchal and M. Siegert, "Ferroelectric thin films for optical applications," Integrated Ferroelectrics, vol. 35, no. 1-4, pp. 1-10, 2001.

[9] D. Mahgerefteh and J. Feinberg, "Shallow traps and the apparent sublinear photoconductivity of photorefractive barium titanate," Modern Physics Letters B, vol. 5, no. 10, pp. 693-700, 1991.

[10] J. Xu, W. Zhang, and Z. Yang, "An optical humidity sensor based on Ag nanodendrites," Applied Surface Science, vol. 280, pp. 920-925, 2013.

[11] X. Wang and X. Liu, "Self-assembled synthesis of Ag nanodendrites and their applications to SERS," Journal of Molecular Structure, vol. 997, no. 1-3, pp. 64-69, 2011.

[12] L. K. Templeton and J. A. Pask, "Formation of $\mathrm{BaTiO}_{3}$ from $\mathrm{BaCO}_{3}$ and $\mathrm{TiO}_{2}$ in Air and in $\mathrm{CO}_{2}$," Journal of the American Ceramic Society, vol. 42, no. 5, pp. 212-216, 1959.

[13] A. Beauger, J. C. Mutin, and J. C. Niepce, "Synthesis reaction of metatitanate $\mathrm{BaTiO}_{3}$-part 2 Study of solid-solid reaction interfaces," Journal of Materials Science, vol. 18, no. 12, pp. 3543$3550,1983$.

[14] B. A. Hernandez, K.-S. Chang, E. R. Fisher, and P. K. Dorhout, "Sol-gel template synthesis and characterization of $\mathrm{BaTiO}_{3}$ and $\mathrm{PbTiO}_{3}$ nanotubes," Chemistry of Materials, vol. 14, no. 2, pp. 480-482, 2002.

[15] G. Pfaff, "Sol-gel synthesis of barium titanate powders of various compositions," Journal of Materials Chemistry, vol. 2, no. 6, pp. 591-594, 1992.

[16] T. Hoffmann, T. Doll, and V. M. Fuenzalida, "Fabrication of $\mathrm{BaTiO}_{3}$ microstructures by hydrothermal growth," Journal of the Electrochemical Society, vol. 144, no. 11, pp. L292-L293, 1997.

[17] P. K. Dutta, R. Asiaie, S. A. Akbar, and W. Zhu, "Hydrothermal synthesis and dielectric properties of tetragonal $\mathrm{BaTiO}_{3}$," Chemistry of Materials, vol. 6, no. 9, pp. 1542-1548, 1994.

[18] M. P. Pechini, "Barium titanium citrate, barium titanium and processes for producing same," Patent US 3231328, 1996.

[19] S. Wada, M. Narahara, T. Hoshina, H. Kakemoto, and T. Tsurumi, "Preparation of nm-sized $\mathrm{BaO}_{3}$ particles using a new 2-step thermal decomposition of barium titanyl oxalate," Journal of Materials Science, vol. 38, no. 12, pp. 2655-2660, 2003.

[20] J.-G. Kim, J.-G. Ha, T.-W. Lim, and K. Park, "Preparation of porous $\mathrm{BaTiO}_{3}$-based ceramics by high-energy ball-milling process," Materials Letters, vol. 60, no. 12, pp. 1505-1508, 2006.

[21] Y. Hotta, K. Tsunekawa, T. Isobe, K. Sato, and K. Watari, “Synthesis of $\mathrm{BaTiO}_{3}$ powders by a ball milling-assisted hydrothermal reaction," Materials Science and Engineering A, vol. 475, no. 1-2, pp. 12-16, 2008.

[22] V. Vinothini, P. Singh, and M. Balasubramanian, "Synthesis of barium titanate nanopowder using polymeric precursor method," Ceramics International, vol. 32, no. 2, pp. 99-103, 2006.

[23] S. Ghosh, S. Dasgupta, A. Sen, and H. S. Maiti, "Synthesis of barium titanate nanopowder by a soft chemical process," Materials Letters, vol. 61, no. 2, pp. 538-541, 2007.

[24] Y. J. Jung, D. Y. Lim, J. S. Nho, S. B. Cho, R. E. Riman, and B. Woo Lee, "Glycothermal synthesis and characterization of tetragonal barium titanate," Journal of Crystal Growth, vol. 274, no. 3-4, pp. 638-652, 2005.
[25] A. V. Ragulya, O. O. Vasylkiv, and V. V. Skorokhod, "Synthesis and sintering of nanocrystalline barium titanate powder under nonisothermal conditions. I. Control of dispersity of barium titanate during its synthesis from barium titanyl oxalate," Powder Metallurgy and Metal Ceramics, vol. 36, no. 3-4, pp. 170175, 1997.

[26] J. Bera and D. Sarkar, "Formation of $\mathrm{BaTiO}_{3}$ from barium oxalate and $\mathrm{TiO}_{2}$," Journal of Electroceramics, vol. 11, no. 3, pp. 131-137, 2003.

[27] W. S. Claubaugh, E. M. Swiggard, and R. Gilchrist, "Preparation of barium titanyl oxalate tetrahydrate for conversion to barium titanate of high purity," Journal of Research of the National Bureau of Standards, vol. 56, no. 4, pp. 289-291, 1956.

[28] M. Stockenhuber, H. Mayer, and J. A. Lercher, "Preparation of barium titanates from oxalates," Journal of the American Ceramic Society, vol. 76, no. 5, pp. 1185-1190, 1993.

[29] M. Z. C. Hu, G. A. Miller, E. A. Payzant, and C. J. Rawn, "Homogeneous (co)precipitation of inorganic salts for synthesis of monodispersed barium titanate particles," Journal of Materials Science, vol. 35, no. 12, pp. 2927-2936, 2000.

[30] W. Lu, M. Quilitz, and H. Schmidt, "Nanoscaled $\mathrm{BaTiO}_{3}$ powders with a large surface area synthesized by precipitation from aqueous solutions: preparation, characterization and sintering," Journal of the European Ceramic Society, vol. 27, no. 10, pp. 31493159, 2007.

[31] K. M. Hung, W. D. Yang, and C. C. Huang, "Preparation of nanometer-sized barium titanate powders by a sol-precipitation process with surfactants," Journal of the European Ceramic Society, vol. 23, no. 11, pp. 1901-1910, 2003.

[32] A. Testino, M. T. Buscaglia, M. Viviani, V. Buscaglia, and P. Nanni, "Synthesis of $\mathrm{BaTiO}_{3}$ particles with tailored size by precipitation from aqueous solutions," Journal of the American Ceramic Society, vol. 87, no. 1, pp. 79-83, 2004.

[33] J. M. Bind, T. Dupin, J. Schafer, and M. Titeux, "Industrial synthesis of coprecipitated $\mathrm{BaTiO}_{3}$ powders," Journal Metals, vol. 39, no. 8, pp. 60-61, 1987. 

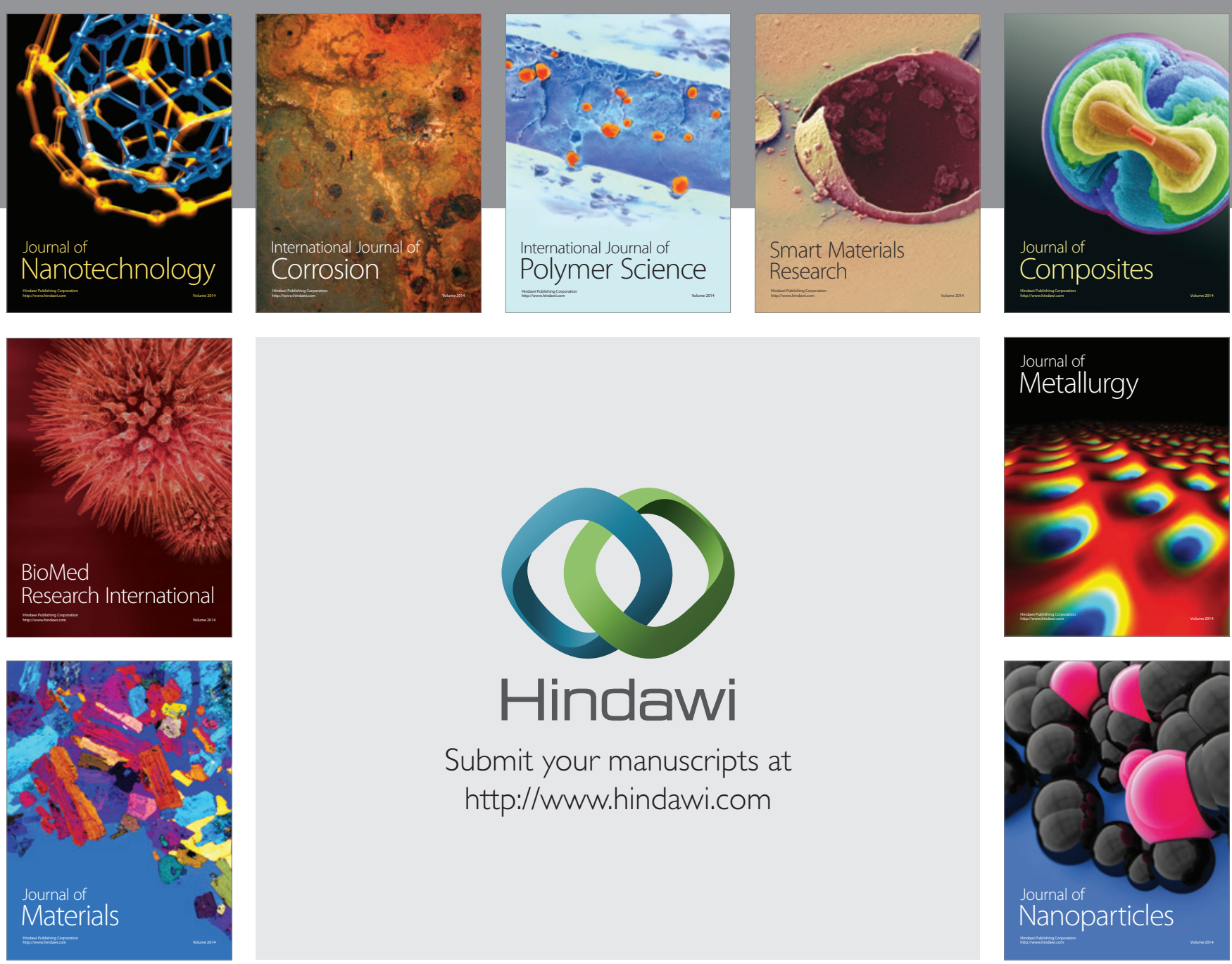

Submit your manuscripts at http://www.hindawi.com
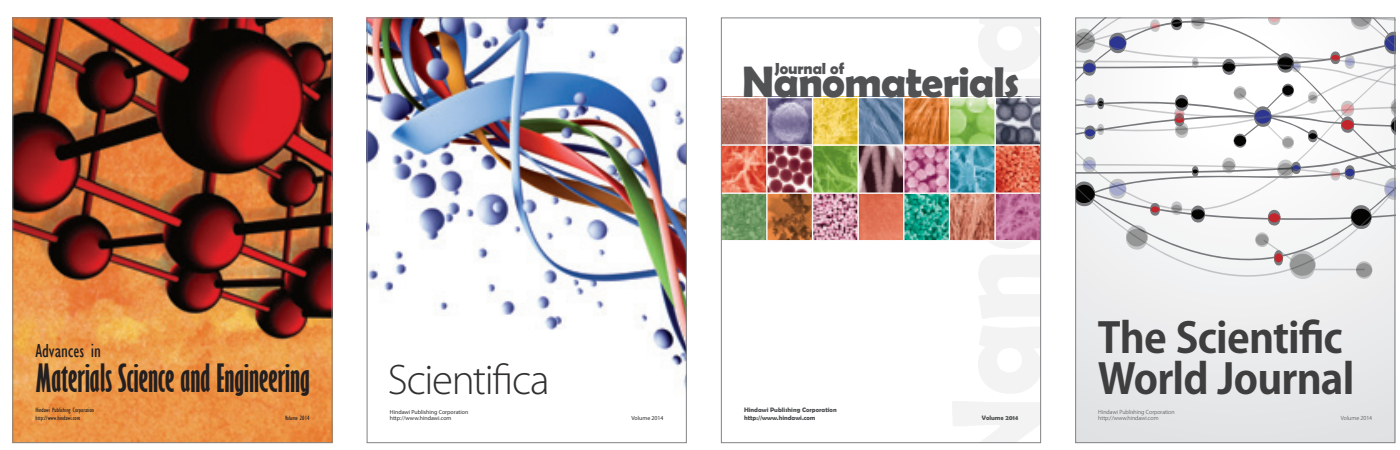

\section{The Scientific World Journal}
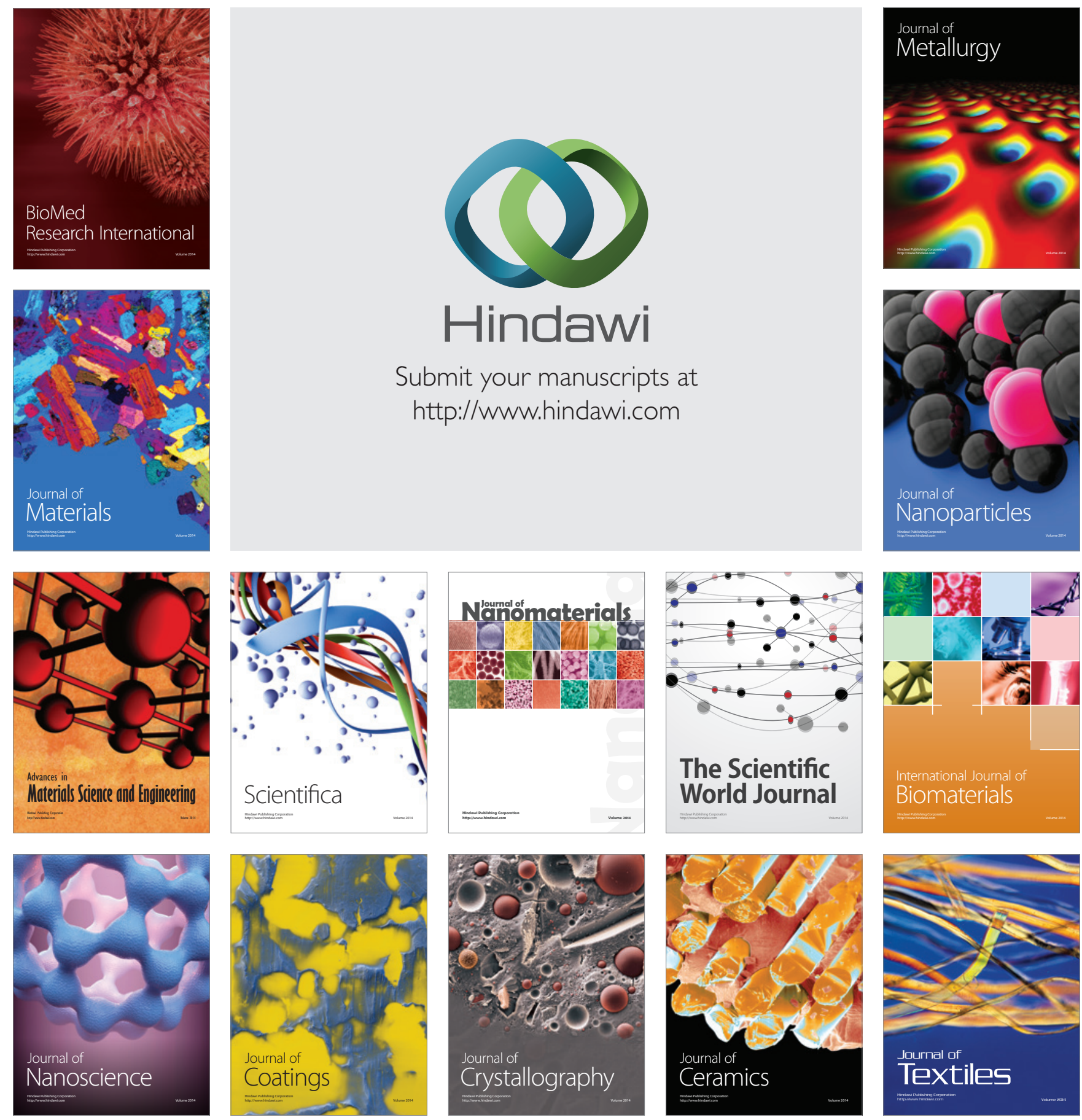